\author{
MILITARY TECHNICAL COLLEGE \\ CAIRO - EGYPT
}

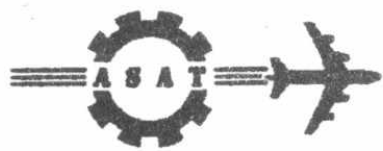

$7^{\text {th }}$ INTERNATIONAL CONF. ON AEROSPACE SCIENCES \& AVIATION TECHNOLOGY

\title{
AIRCREW COMBAT TRAINING SYSTEM SIMULATION
}

\author{
M. A. Matar* \\ H. M. S. Abdel-Wahab* \\ W. M. Tawfeek*
}

\section{ABSTRACT}

Military training systems are continuously evolving to replicate the real world conditions and to afford the advantage of real situation (live) training through sophisticated electronic equipment. Such aircrew training systems are introduced to improve the aircrew proficiency and to enable them to recognize weapon envelope boundaries and repeatedly observe the results of the simulated firing against manned high performance aircraft under certain controlled conditions.

A training system that incorporates the real aircraft themselves should comprise a tightly linked borne segment and a ground segment. The borne segment records flight and weapon data and transmits them to the ground upon request. The ground segment comprises a tracking subsystem and a control-and-computation (CCS)subsystem leading to a display and debriefing (DDS)subsystem. The transmitted data by the borne segment is to be received by the tracking subsystem and relayed to the CCS. The CCS employs a master computer to compile, record, process and display the data on the multi-color video displays of the DDS for monitoring the flight in progress and later replaying during debriefing .

A complete real time simulation of aircrew training system is currently being conducted in the M.T.C. This work is developed using transputer-based processing elements where parallel data processing techniques are adopted in order to speed up the simulated system operation to be close to the real system. This paper presents a simulation of the computation and display functions (processes) as implemented in the actual training system, where multiple aircrafts are concurrently (simultaneously) tracked and their locations are recorded and displayed throughout the exercise.

The simulation of such functions serves as preliminary exposure to the actual training systems for both the trainer and the trainee. Moreover, it would facilitate the system adaptation to various aircraft (F-16, Mirage ..etc.) in conjunction to various weapons (Bombing and missile missions, with accurate simulation without using live ammunition).

Keyrvords : Aircrew combat training .simulation. parallel processing, transputers ,transponder, aircraft.

* Avionics Department, M.T.C., Cairo. 


\section{INTRODUCTION}

Air Combat Maneuvering Instrumentation system [1] is an air combat training system. It is used to train and improve the fighter aircrew maneuvering actions and firing proficiency when they are in the air combat.

Such trainers are operated in real flight environment. The trainees, within the training area, perform all flight maneuvers, target acquisition and tracking activities. The sole deviation from the real combat is that a weapon simulation is used. Thus a scoring and monitoring system is needed.

The monitoring functions entails a data link through which all flight information (that one not attainable by radar) are communicated to the monitoring and scoring center. A secondary radar function is needed to locate the $\mathrm{a} / \mathrm{c}$ within the battle field.

Thus the air combat training system comprises [1]

- An airborne segment to collect and communicate flight information to the ground. To make the system platform independent, a pod with the necessary instrumentation is used. A link with $\mathrm{a} / \mathrm{c}$ attack avionics is required at least for signalizing target lock-on and fire instants. A secondary radar function is to be supplemented.

- Monitoring center with control and radio transmission-reception functions. Transmission and reception function requires implementation of several synchronized interrogation for secondary radar functions and usually centralized reception station, known as master station. Management of the interrogators is done by such a master station.

Since master station is generally located at the center of the training area, an additional link is required to relay the collected information to the airbase-located monitoring center. Apart from the radio frequency links, the control and computation of both the aircraft and target tracking and weapon simulation functions are to be performed. Moreover a display subsystem is needed for visualizing and evaluating training session in progress and for afterthe-action debriefing.

A typical functional diagram for the complete air combat training system is shown in Fig. 1 [1]. The CCS initiates the master station which, in turn, initiates the selected interrogator for transmission. The borne pod of selected ID is triggered to release the following information:

- Flight data ( air speed, heading angle, elevation angle, altitude).

- Ranging data (distances between the aircraft and the remote stations).

- Firing data (radar information, lock-on, firing instant).

This information is received from the master station (base band communication) to compute the aircraft position, velocity, acceleration and the attitude corrections to transmit to that aircraft. Then it transmits the aircraft position, speed, attitudes, lock status and its ID to the display. Then it checks the firing pulse if exists, it trigger the missile simulation program, supplying this program with all information about the target to track, give the firing result, and transmits the position, heading of the missile and firing result to be displayed. DDS receives information from the CCS. This information can be either about the aircraft or the missiles and is displayed on graphic screen(aircraft and missile) or data screen(aircraft only). 


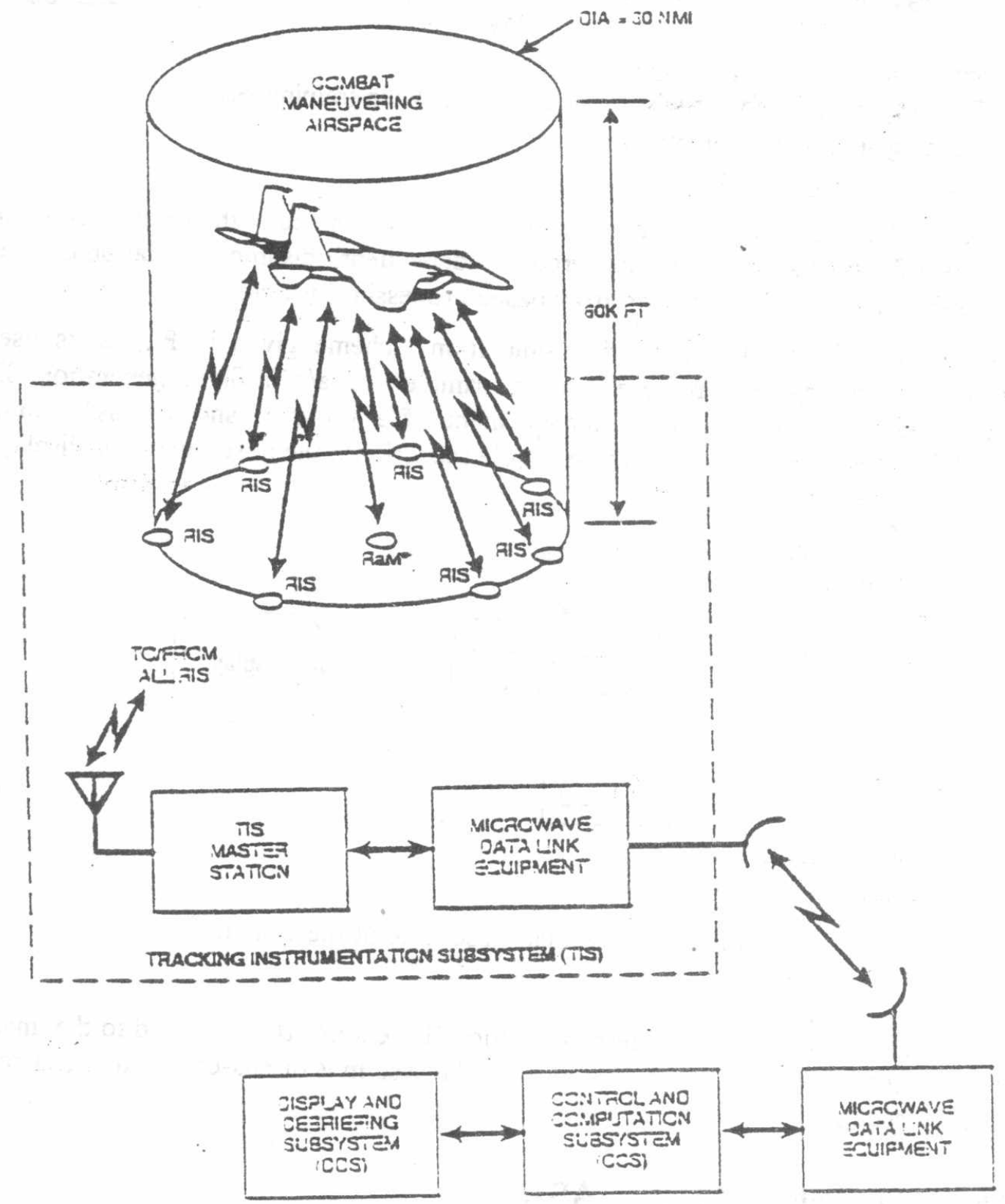

Legend

RIS - Remote Interrogation

Ra.l- Remote-at-Master Co-located with Vaster Station

Figure 1 tir Combat Vaneuvering Instirumentation Sistem 
The present work has the following tasks:

- Target generation for simulating aircrafts' tracks in training session.

- Training session management.

- Display of target $(\mathrm{a} / \mathrm{c})$.

The implementation of such a system in real time is one of the main aims of this work. Since, most of these tasks can be considered as independent, this implementation is investigated within the framework of a transputer-array based processing system.

To attain these objectives the simulation scheme given in Fig. 2. is used. Two interconnected computers are used as a sample of a/c in-flight generation. Data are communicated to transputers under management of a program and a missile simulation is generated. Transputer system displays the whole scenario on the host computer display.

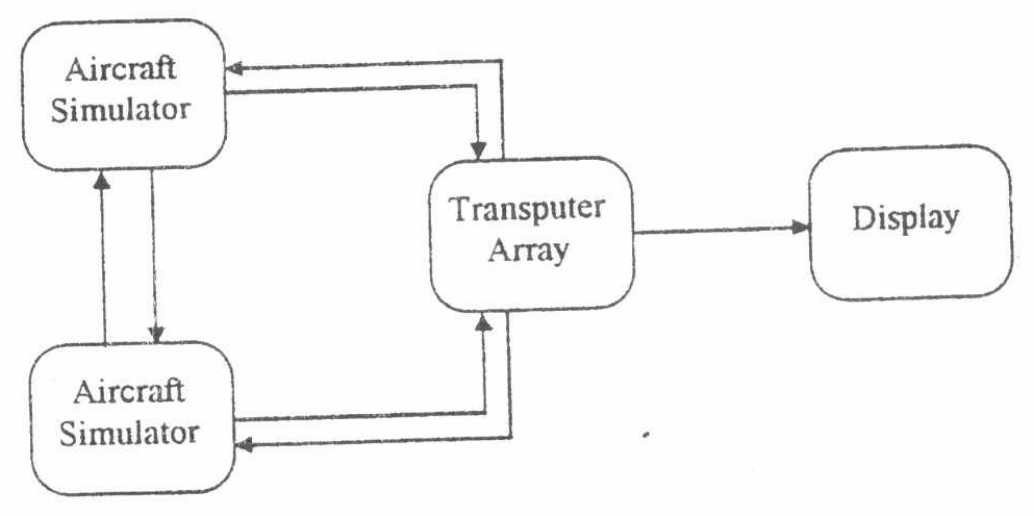

Figure 2: Simulation scheme of the system

Target generation is considered in section II. Section III is devoted to the management problem. In section IV, the display function of the system is discussed. Results and conclusions are given in section $\mathrm{V}$.

\section{TARGET GENERATION TASK}

This task is concerned with the simulation of controlled a/c motion as well as the simulation of the a/c Head Up Display (HUD).

\section{a) Aircraft Motion}

The aircraft motion simulation is based upon representing the aircraft by a point in space with coordinates $(x, y, z)$. This point moves with speed $V$ in direction making an angle $\alpha$ with the north (y-axis) and elevation angle $\beta$ as shown in Fig. 3. The aircraft motion can be considered as a translation (moving the $\mathrm{a} / \mathrm{c}$ ahead) and rotation (in the three directions around its axes). Making Yaw rotation results a change in the heading of the aircraft i.e. making angle $\propto$ with the north axis (y-axis) as shown in Fig. 3. Pitch variation yields ascent or descent of the $\mathrm{a} / \mathrm{c}$ and can be viewed with variation of the elevation angle $\beta$. 


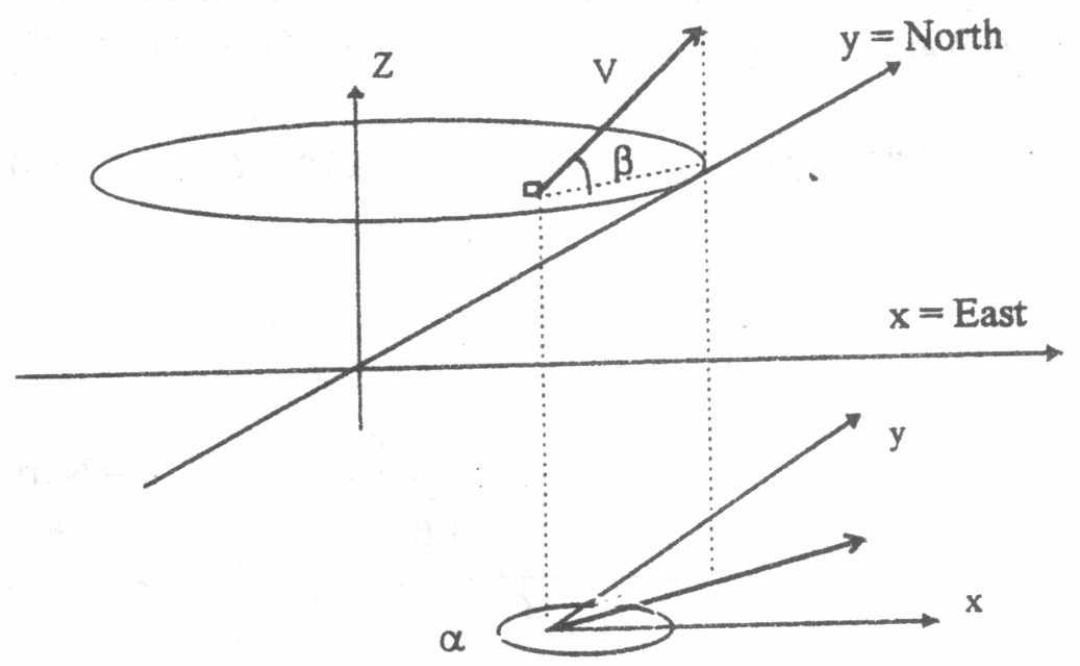

Figure 3: Geometrical decomposition of a point moving with speed V, with heading $\alpha$, and elevation $\beta$.

An example of translation combined with YAW rotation is shown in Fig. 4. Let Co be the starting point and the aircraft is assumed to move with heading $\alpha$ and speed $\mathrm{V}$ for time $\mathrm{t}$. The a/c will reach $\mathrm{Cl}$ given by [2] :

$$
\vec{C}_{1}=\vec{C}_{0}-V t \sin (\alpha) \vec{x}+V t \cos (\alpha) \vec{y}
$$

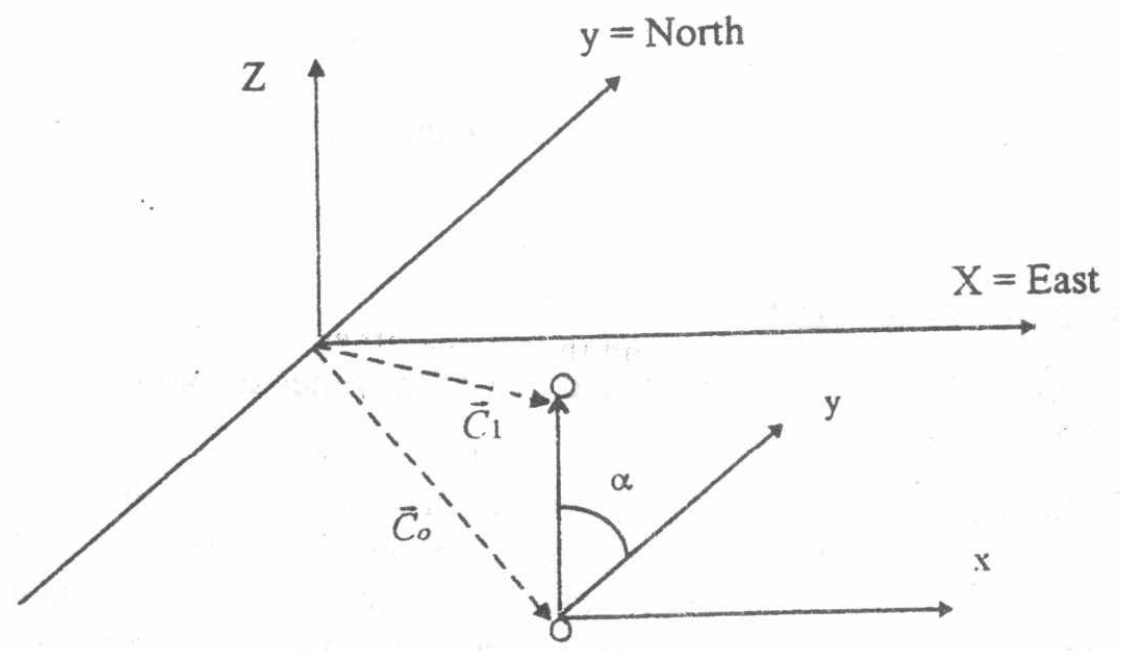

Figure 4: A translation and rotation with $\alpha$ to the north. 
A more general case is given in Fig. 5, where the a/c moves with heading angle $\alpha$, elevation angle $\beta$ and speed $V$ for a time t. This motion can be described by [2]:

$$
\vec{C}_{1}=\vec{C}_{o}-V t \sin (\alpha) \cos \beta \vec{x}-V t \cos (\alpha) \cos \beta \vec{y}+V t \sin \beta \vec{z}
$$

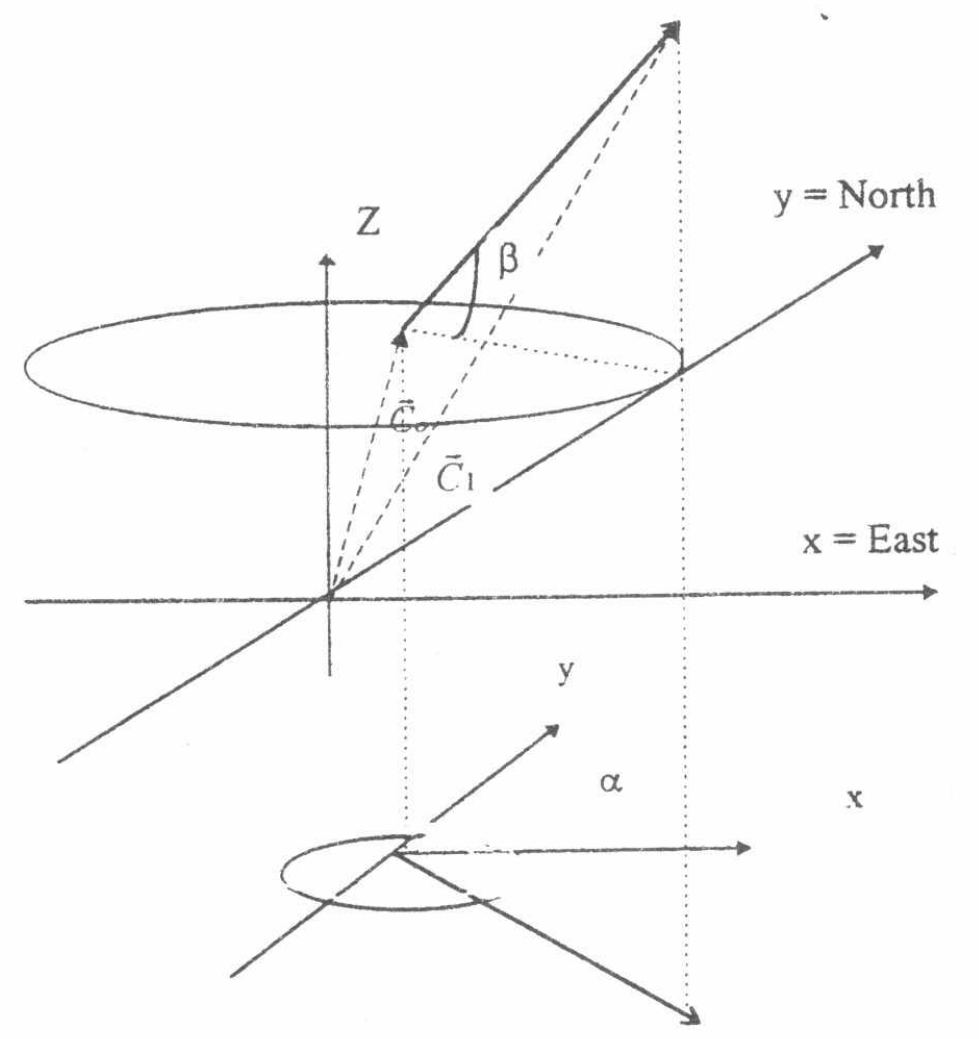

Figure 5: A motion with angle $\beta$ with the north and elevation angle $\alpha$.

\section{b) Simulation of aircraft HUD}

The information of the aircraft and that of the other air targets are displayed on the PC monitor simulating the HUD.

All flight parameters of the aircraft such as altitude, heading, pitch up, pitch down, speed. as well as range of the target, are displayed superimposed either on the $3-\mathrm{D}$ real world view mode or on the 2-D air-to-air combat mode. In the 3-D real world view, the a/c can view a cone in which it represents its vertex and targets are considered as a set of spheres. In the airto-air combat mode, 2-D view in the $x-y$ plane is obtained. The dimension of this $x-y$ plane is $15 \mathrm{Km} \mathrm{X} 15 \mathrm{Km}$ and centered at the location of the aircraft, a circle symbol represents a target aircraft location in $x-y$ plane. Figure 6 , for example, shows the HUD view during training session of two $\mathrm{A} / \mathrm{Cs}$ in the air-to-air combat mode.

In this simulation, HLD screen in the air-to-air mode is updated through data exchanging between the two aircraft as a simulation of a/c radar. Using a serial/parallel links data is 

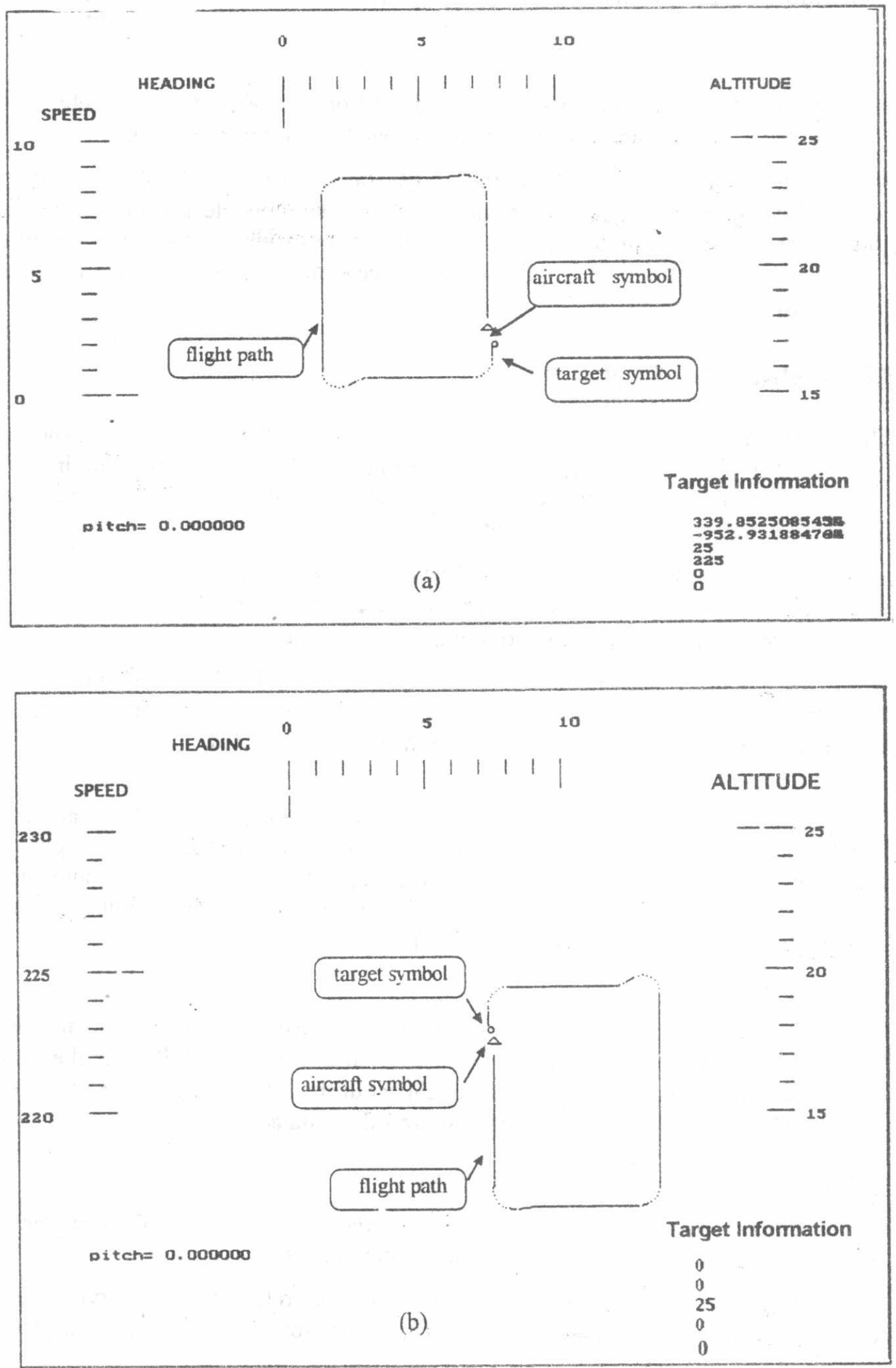

Figure 6: HUD View during training session of two $\mathrm{A} / \mathrm{Cs}$ in the air-to-air combat mode. (a) Aircraft 1 HUD screen $\quad$ (b) Aircraft 2 HUD screen 
transferred between the two computers on the serial port or parallel port respectively. This data is ( $\mathrm{a} / \mathrm{c} \mathrm{ID}$, air speed, heading angle, elevation angle, altitude, aircraft position).

As to guided missile simulation, track is generated as in the case of an aircraft except that the heading angle $\alpha$ and elevation angle $\beta$ of the tracking missile are computed along the target flight path and firing result is given. It should be mentioned here that, full control by the trainee upon his $\mathrm{a} / \mathrm{c}$ is provided (pitch, heading, acceleration, firing missiles, ...etc.) through simple key strokes.

\section{MANAGEMENT FUNCTION}

The management of the system operational cycles is allocated to the transputer array shown in Fig. 2. This is done on a polling (or interrogation)basis. The transputer interrogates an a/c among The group of simulated flying aircraft through a group of processes. This initiates computers to dump the following information:

- Flight data ( air speed, heading angle, elevation angle, altitude,).

- Ranging data (distance between the aircraft and the reference origin).

- Firing data ( radar information, lock-on, firing instant).

and passes the required information (absolute position and heading angle) to the display. These information are also used as initial condition for missile simulation function when needed. Then missile simulation data is passed to the display if needed.

In real system, several processes occur concurrently, the sequential interrogation to each aircraft, $\mathrm{a} / \mathrm{c}$ maneuvers, missile launching $\ldots$ etc. To realize these functions by a computer, these functions have to be executed concurrently. This suggests the use of transputer for their implementation. Moreover, the $\mathrm{a} / \mathrm{c}$ speeds, and the need to approach full motion video displaying entail the use of parallel processing facility.

Figure 7, depicts a message passing architecture used to implement the management function, where two Transputers TrO \& Trl carry out the display function for the aircraft or missile and the third Transputer Tr2 is used to carry out the interrogation (or Server) Process and the Router Process and acquisition of $\mathrm{N}$ aircraft flight data.

\section{In the following, we outline the processes executed:}

- Server process transmits to the Router process the selected aircraft ID, and waits for reception after the $\mathrm{a} / \mathrm{c}$ interrogation by the Router process.

- The required $\mathrm{a} / \mathrm{c}$ transmits its frame to the Router that relays it to the Server which in turn passes it to the display function through transputer links so as to display the aircraft or missile if needed.

- The Server repeats the whole process as far as the training session continues. 


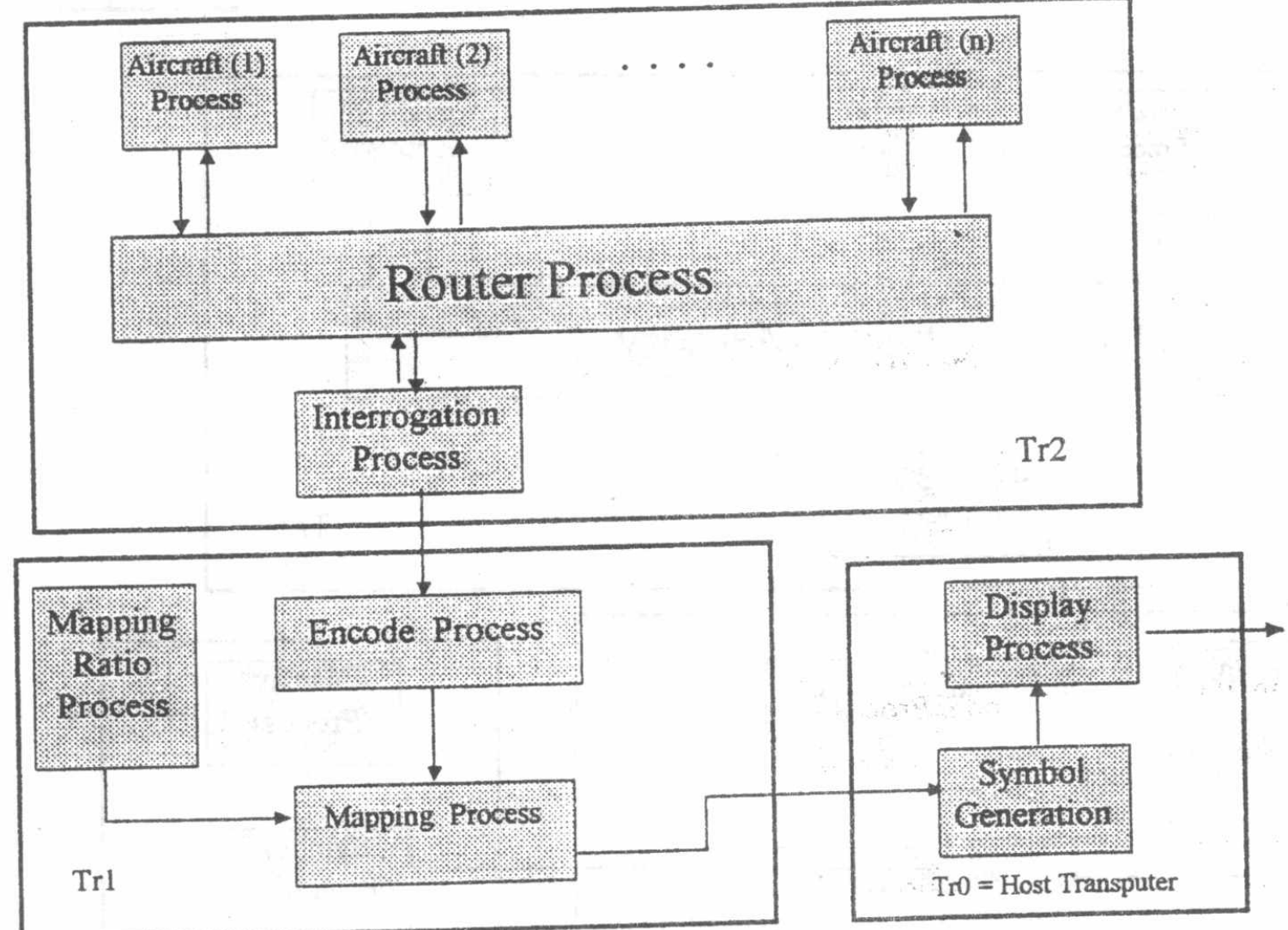

Figure 7: The Message passing architecture implementation Management Function.

Several "Scenarios" are examined on the basis of message passing data exchange mechanism between the $A / C$ process and the router process. These scenarios are:

1 - A/C process tests whether or not an interrogation is done by the Router process after each computation of $\mathrm{a} / \mathrm{c}$ position. If an interrogation is sensed it passes the required information, then continues to the new position computation. This is denoted by "Scenario 1" on Figure 11.

2 - A/C process suspends for a defined time after each position computation, then tests for interrogation to reply otherwise continues to the new position computation. This is denoted by "Scenario 2" on Figure 11.

3 - A/C process waits after each computation of $\mathrm{a} / \mathrm{c}$ position until receiving and then replying the interrogation. This is denoted by "Scenario 3" in Figure 11.

Evaluating these three scenarios on the basis of a time performance index, has led us to consider the shared variable implementation depicted in Fig. 8. In Fig. 8, a shared memory variable is used to interface the $\mathrm{a} / \mathrm{c}$ process to the server process. This allows the $\mathrm{a} / \mathrm{c}$ processes to save their data each position computation cycle to this memory and continues to the new position computation after a pre-defined time. Except for this change, the system operates as explained before. This is considered as the "Scenario 4" as shown in Figure 11. 


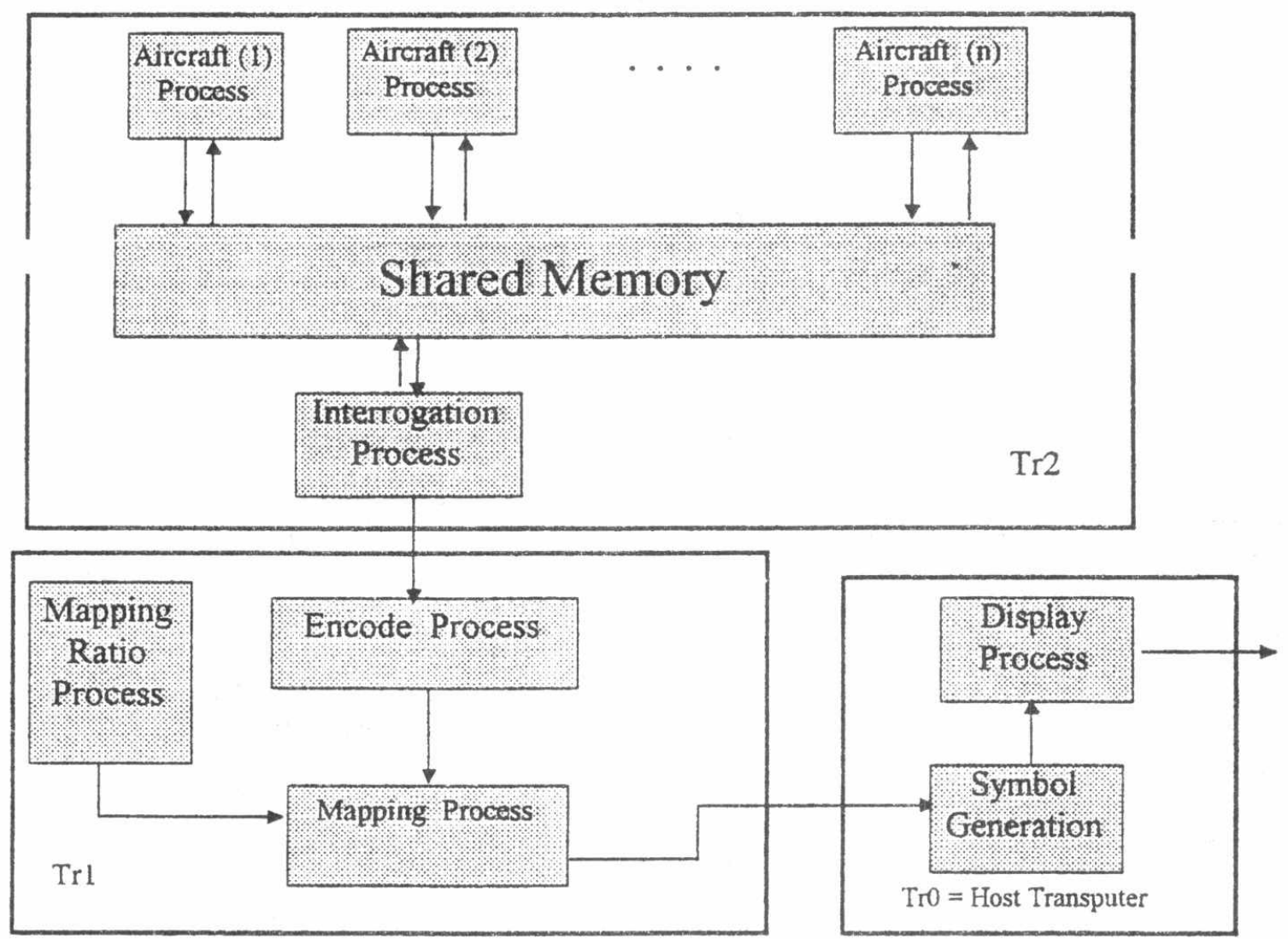

Figure 8: Shared variable implementation of Management Function as used in Scenario 4

\section{Display function}

This function is intended mainly for graphical representation of target and missile trajectories on the DDS. Mapping of actual position in real world onto display entails scaling and coding. Scale factors are denoted by : Sx, Tx, Sy and Ty are[3] :

$$
\begin{aligned}
\mathrm{Sx} & =(\mathrm{Vr}-\mathrm{Vl}) /(\mathrm{Wr}-\mathrm{Wt}) \\
\mathrm{Sy} & =(\mathrm{Vt}-\mathrm{Vb}) /(\mathrm{Wt}-\mathrm{Wb}) \\
\mathrm{Tx} & =(\mathrm{Vl} \mathrm{Wr}-\mathrm{Wl} \mathrm{Vr}) /(\mathrm{Wr}-\mathrm{Wt}) \\
\mathrm{Ty} & =(\mathrm{Vb} \mathrm{Wt}-\mathrm{Wb} \mathrm{Vt}) /(\mathrm{Wt}-\mathrm{Wb})
\end{aligned}
$$

where $\mathrm{Vt}, \mathrm{Vb}, \mathrm{Vr} \& \mathrm{Vl}$ represent the Display coordinate, and $\mathrm{Wt}, \mathrm{Wb}, \mathrm{Wr} \& \mathrm{Wl}$ represent the Real World coordinate.

The scale factors are calculated in the Mapping Ratio Process (Fig. 7 \& Fig. 8). The position $(\mathrm{x}, \mathrm{y})$ is checked whether or not it lies inside the display screen and will be displayed after making the necessary mapping

$$
\begin{aligned}
& \text { C. } \mathrm{I}=\mathrm{x}<\mathrm{Wl} ; \\
& \text { C. } \mathrm{r}=\mathrm{x}>\mathrm{Wr} ; \\
& \text { C. } \mathrm{t}=\mathrm{y}<\mathrm{Wt} ;
\end{aligned}
$$


$C \cdot b=y>W b$

if $C . r=C . t=C . b=C .1=$ FALSE this point lays inside the display screen and it should be displayed [3]. This is done by the Encode Process..

The Mapping Process takes the position in the real world coordinates $(x, y, z)$ and uses the previously-defined scaling factors to obtain the display coordinates $(\mathrm{x} 1, \mathrm{yl}, \mathrm{zl})$ for presentation on the screen. The following equation shows such transformation [3]:

$$
\begin{aligned}
& x 1=x \cdot S x+T x \\
& y 1=y \cdot S y+T y
\end{aligned}
$$

Apart from the trajectory displaying, symbols for the for target and missiles are displayed.These symbols are generated and their attitude are matched to their real parameters by the transputer. Then ,these symbols are superimposed upon the respective trajectories.

Figure 9 shows a flowchart for the processes included in the display function. The display function is implemented on two transputers in a pipeline topology). Transputer 0 is dedicated to implement the symbol generation function. As to the encode and mapping processes transputer 1 is used. Transputer 1 receives the information from transputer 2 (as discussed in the previous section ), carries out the encode, mapping processes and transmits target. information to transputer 0 , which in turn, superimposes the generated symbol and relays the data to display.

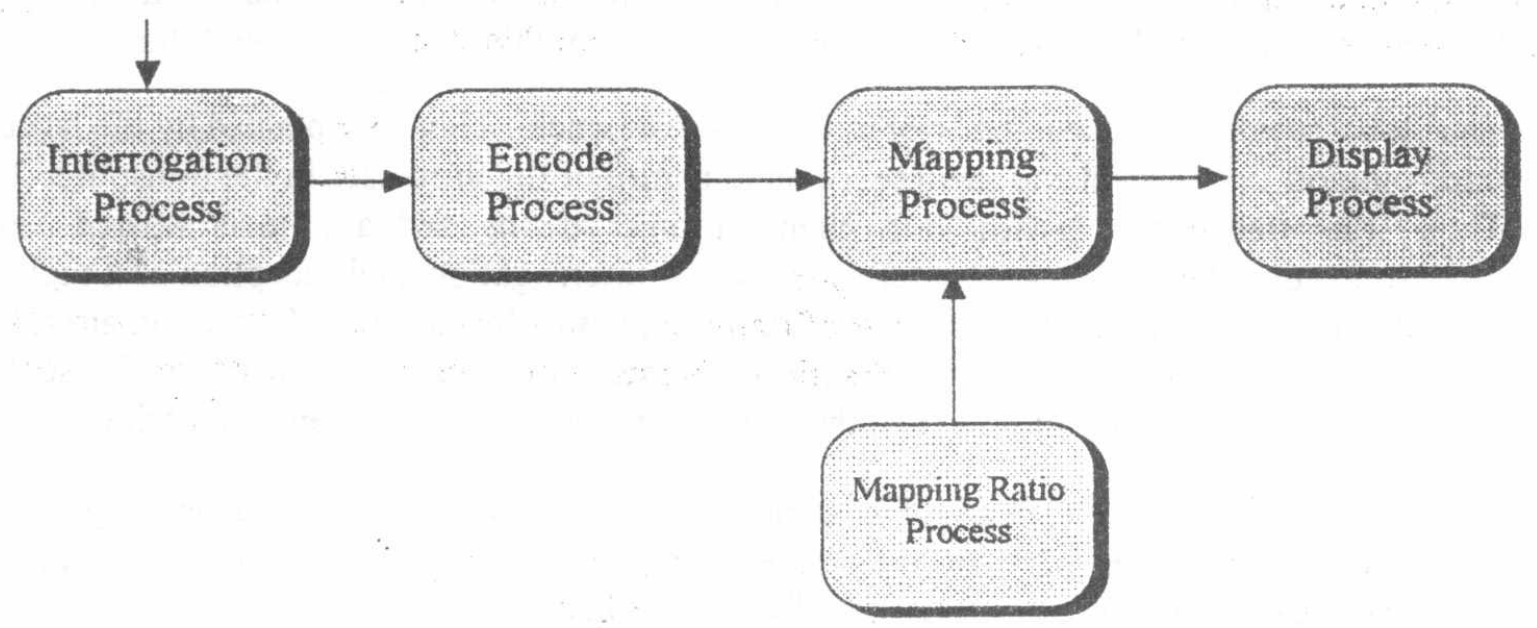

Figure 9: Process Flowchart as included in the Display Function

\section{Results and Conclusions}

The hardware configuration adopted in this work was built around the INMOS T800 Transputer. All the above mentioned tasks were developed on IBM PC-AT with the TRAM[5]. This comprises one host transputer with 4 Mbytes RAM and another two transputers with 2 Mbytes RAM each. Each of these transputers can communicate with the others through one or more of its four serial links, (see Fig. 10). As to the parallel processing software, it was developed using the Parallel C language from $3 \mathrm{~L}$ system [6]. 


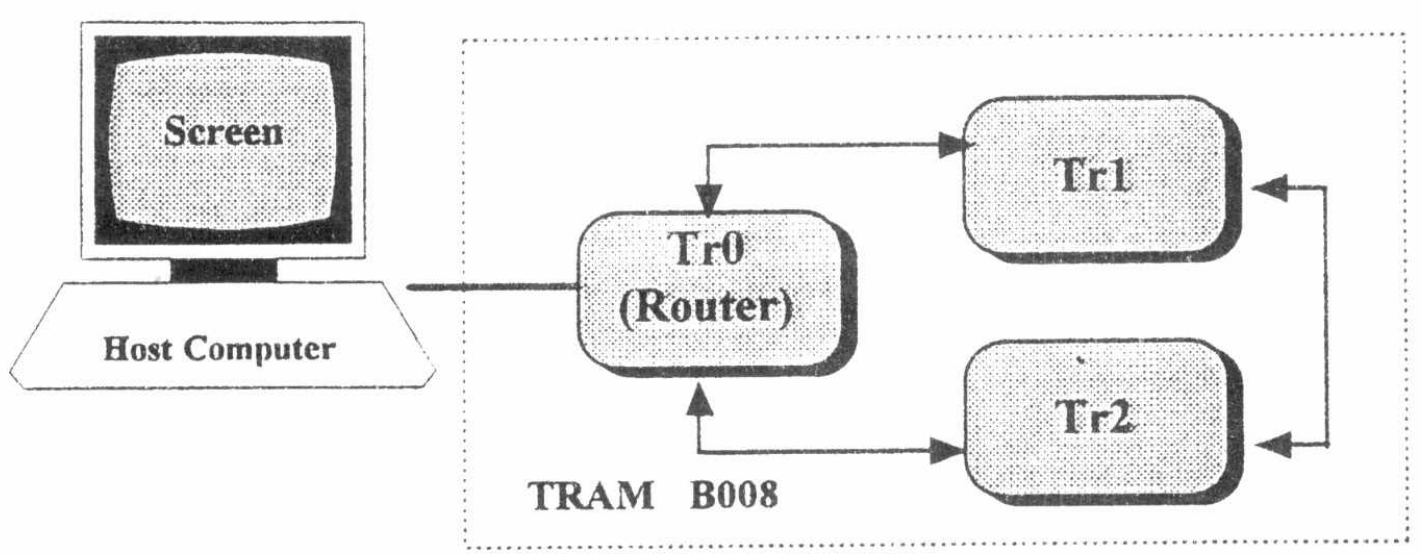

Figure $10:$ The hardware development tools used in the study

To asses the system performance we consider the following indices:

1- Time performance in relation to the real system operation

2- System expandability to $\mathrm{N}$ target working in pairs or in other formation

As to the first index, Fig. 11 shows the time performance for the various configuration introduced in section 3 on the basis of Fig 7 and Fig. 8. From this graph, it is found that :

A - The 1st scenario yields the worst time performance. This is explained by the large interrogation time period and the free running nature of the processes in the transputer 2. Thus, the transputer carries out them in time sharing and a time is wasted in synchronization between the Router process for interrogation and reception of a reply. To use this configuration the number of transputers must be increased (for use in aircraft processes) so as to reduce the time sharing processes in one transputer. In such conditions, this configuration will be the best one for use in such system simulation.

B - The 2nd scenario suffers nearly the same problems of the first one. Suspending each process by transputer 2 for a given time doesn't improve significantly the time sharing process even with the reduction of the interrogation period.

C - The 3rd scenario yields the best time performance while keeping the same architecture (Fig. 7.). This is explained by the smaller the interrogation time period. However, it has a basic disadvantage of constrained (not free running) aircraft processes. This means that for every computation cycle for each process, the computation process waits for an interrogation to be replied to the Router before advancing to the second computation cycle. This limits the time sharing process in the transputer 2 to only 2 processes at most and hence results in reducing the interrogation time period.

D - The fourth scenario (architecture Fig. 8 ) has all processes in a free running mode in transputer 2. To overcome the synchronization problem confronted in architecture of Fig. 7 , a shared memory is used to link the interrogation process and the aircraft processes. This results in best interrogation time period and hence it is recommended for the real time simulation problem whenever the number of transputers is small( as in our case ). 
As to second index, aircrafts are considered at work in pairs, other aircraft formations are currently being examined.

From the considered implementation approaches: message passing and shared variable, the number of transputers and the number of tasks as well as their complexity drive the proper choice of either approach. For small number of transputers ( 3 in this study) the shared memory approach is considered as the proper choice.

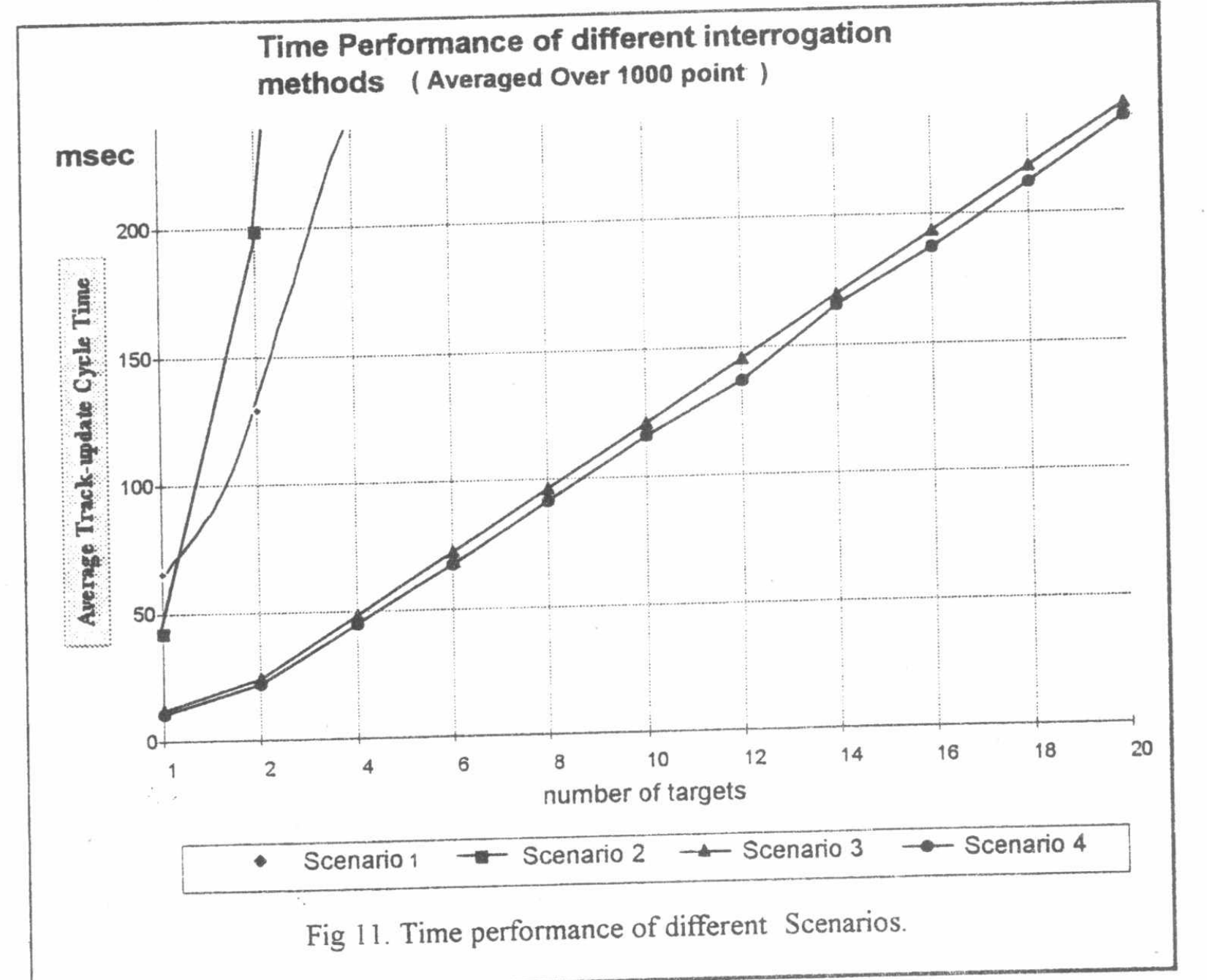

\section{References:}

[1] T.J.Gander “Janes's Military Training System "1991-1992.

[2] F.P.Beer and Russell "Vector Mechanics for Engineers: Dynamics".McGraw-Hill, 1990

[3] F.S.Hill "Computer Graphics", Macmillan Publishing Company, 1990.

[4] O. R. Brookes and A. J.Stewart: 'Introduction to OCCAM-2 on the transputer" Chap. 3, Macmillan Publishing Company, 1989.

[5] Grabam and T. King “The Transputer Handbook", Prentice Hall, N.Y, 1990.

[6] 'Parallel C User Guide ", 3L Systems , 1989. 